\title{
Blow-up Criterion for Compressible Navier-Stokes Equations with Degenerate Viscosities
}

\author{
wenjing song ${ }^{1}$ and Wenhuo $\mathrm{Su}^{2}$ \\ ${ }^{1}$ Xi'an Polytechnic University \\ ${ }^{2}$ Yichun University
}

January 28, 2022

\begin{abstract}
In this paper, a blow-up criterion of classical solutions to the Cauchy problem for the compressible Navier-Stokes equations with degenerate viscosities is established, as well as the Shallow water equations. Moreover, we prove that the maximum norm of the gradient of velocity controls the possible breakdown of regular solutions for compressible Navier-Stokes equations with degenerate viscosities.
\end{abstract}

\section{Hosted file}

manuscript.pdf available at https://authorea.com/users/457734/articles/554451-blow-upcriterion-for-compressible-navier-stokes-equations-with-degenerate-viscosities 